\title{
Analysis of contamination of blood cultures in a tertiary care hospital in Sri Lanka
}

\author{
KD Namalie, WHS De Silva, FS Makeen, N Senevirathna, AN Pallegoda
}

\section{Introduction}

Blood culture is one of the important investigations performed in the microbiology laboratory to find the aetiological agents in patients presenting with sepsis syndrome. However, contamination is a common pre-analytical error which compromises the utilization of this useful test. The accepted standard is to keep blood culture contamination rates less than $3 \%$. Inadequate evaluation of blood culture contamination events can affect patient management. Therefore, this study was done to evaluate blood culture contamination rates and to analyze contaminants according to the organism, patient location and prior antibiotic use at a tertiary care hospital.

\section{Methods}

The study was carried out in the microbiology laboratory, Colombo North Teaching Hospital where BacT/ALERT system is used for blood culture. Blood cultures received from 01/01/2019 to 31/12/2019 were analyzed retrospectively using WHONET software. Details were gathered by informing all blood culture positives to the relevant clinicians and following factors were considered to decide whether a particular isolate is clinically significant or a contaminant.

1. Identity of the organism

3. Time to positivity
2. Number of blood cultures positive out of blood cultures received for the same organism

4. Clinical details of the patient

The microbiologist and the clinician together took decisions, taking into account the clinical parameters of the patient such as the presence of prosthetic devices and immunocompromised status.

\section{Results}

The total number of blood cultures received was 7819, of which 1092 were signaled positive by the system. Of these positive cultures, 438 (5.6\%) were identified as contaminants.

Coagulase negative staphylococcus (61.9\%), Bacillus spp. (7.5\%), Corynebacterium spp. (3.2\%), Acinetobacter spp. (1.6\%), Enterococcus spp. (0.5\%), Streptococcus constellatus (0.2\%) mixed bacterial growth $(24.4 \%)$, and Staphylococcus aureus $(0.6 \%)$ were identified as contaminants after thorough evaluation of the clinical condition of the patients.

The highest number of contaminants were identified from medical wards (53.6\%). Surgical wards $(6.8 \%)$, paediatric wards $(7.1 \%)$ and surgical and medical intensive care units $(7.9 \%$ and $6.8 \%$ respectively) had similar rates of contamination. The two neonatal units $(3.7 \%$ and $3.4 \%)$ had relatively low rates of contamination.

Of all contaminants, $221(50.4 \%)$ patients received antibiotics prior to collection of blood culture.

\section{Conclusion}

Blood culture contamination rates are higher than accepted rates. Active interventions are needed to correct this.

Keywords: blood culture, blood culture contaminants, contamination rates

Department of Microbiology, Colombo North Teaching Hospital, Ragama, Sri Lanka 